\title{
Clinical Experience of Integrative Autism treatment with a Novel type of Immunotherapy
}

\author{
Nicola Antonucci ${ }^{1}$, Stefania Pacini ${ }^{2 \star}$ and Marco Ruggiero ${ }^{2}$ \\ ${ }^{\prime}$ Biomedical Centre for Autism Research and Treatment, Bari, Italy \\ ${ }^{2}$ Silver Spring sagl, Via Raimondo Rossi 24, Arzo-Mendrisio, Switzerland
}

\section{Article Info}

\section{*Corresponding author:}

Stefania Pacini, MD, PhD

Silver Spring sagl

Via Raimondo Rossi 24

Arzo-Mendrisio 6864

Switzerland

E-mail: info@bravo-europe.com

Received: February 10, 2019

Accepted: February 22, 2019

Published: February 28, 2019

Citation: Antonucci N, Pacini S, Ruggiero M. Clinical Experience of Integrative Autism treatment with a Novel type of Immunotherapy. Madridge J Vaccines. 2019; 3(1): 71-76. doi: 10.18689/mjv-1000116

Copyright: (c) 2019 The Author(s). This work is licensed under a Creative Commons Attribution 4.0 International License, which permits unrestricted use, distribution, and reproduction in any medium, provided the original work is properly cited.

Published by Madridge Publishers

\begin{abstract}
In this study, we report the results associated with a novel type of immunotherapy based on a supplement containing low-molecular-weight chondroitin sulfate, phosphatidylcholine, and vitamin $D_{3}$. In October 2018, the Biomedical Centre for Autism Research and Treatment of Bari, Italy, started using this approach on autistic subjects. By January 2019, several scores of patients have been treated; here, we describe the cases of three autistic patients for whom the treatment was remarkably effective. In order to obtain the most objective and complete evaluation of results, we used the Autism Treatment Evaluation Scale (ATEC), a diagnostic assessment tool that has been demonstrated effective in evaluating interventional effects in autism. Our observations indicate that some of the most representative symptoms of autism were completely normalized after eight weeks of treatment and other showed a trend toward normalization. No adverse effects were reported. Based on the cases described in this report and on our ongoing research, we are convinced that this type of supplementbased immunotherapy can be beneficial to autistic patients and represents a new and promising treatment. We expect that the described approach will play a central role in future treatments for autism, both alone and in combination with other therapies such as behavioral therapies, nutritional interventions or manual lymphatic massage.
\end{abstract}

Keywords: Autism; GcMAF; Immune system; Neuroinflammation; Immunotherapy.

\section{Introduction}

Autism is a complex disorder that involves primarily the development of the brain but is also quite often associated with abnormalities of immune responses and gut function as well as with alterations of microbiota composition. We recently postulated that autism and cancer share a number of similarities at the level of cellular and molecular signal transduction, the involvement of the immune system and of the microbiota [1]. In our opinion, it is not surprising that immunotherapeutic approaches originally developed for cancer may find application in the field of autism. A notable example of such a translation of approaches from cancer to autism is represented by the immunotherapeutic factor termed Gc protein-derived Macrophage Activating Factor (GcMAF). This immunotherapeutic agent proved effective in the context of integrated approaches to cancer as well as in the treatment of autism as demonstrated by a number of independent observation [2-8]. The consideration that one factor is responsible for multiple therapeutic outcomes in different diseases is not surprising if we consider that GCMAF, in addition to balancing the immune system, shows neuroprotective effects [9] with particular reference to microglial cells [10], a type of neuro-immune cells implicated in the pathogenesis of brain diseases such as autism or Alzheimer's disease [11]. 
Based on our extensive research experience with GcMAF since 2011 [12], and on recent molecular evidence on the role of glycosylation of the protein moiety of GcMAF [13], in 2016 we hypothesized that the effects of GCMAF in vitro and in vivo were to be attributed to a glycosaminoglycan, chondroitin sulfate [14]. Consistent with this hypothesis, a supplement based on chondroitin sulfate, proved as effective as GCMAF in improving the symptoms of autism as described in a study published in 2017 [15]. A major limitation common to the GcMAF used in the studies quoted above $[7,8]$ as well as to the supplement based on chondroitin sulfate termed Rerum [15] is represented by the extractive nature of the main active components; GcMAF was of human origin, whereas the chondroitin sulfate in Rerum was from animal origin (bovine cartilage) and was a mixture of heterogeneous low- and highmolecular-weight species. Therefore, in order to overcome the limitation due to the presence of a molecule-chondroitin sulfate - of animal origin and of heterogeneous molecular weight, we designed a novel immunotherapeutic supplement designated imuno ${ }^{\circledR}$ that is an emulsion of microbial (nonanimal-derived), homogeneous low-molecular-weight chondroitin sulfate non-covalently bound to pure phosphatidylcholine with vitamin $D_{3}$ intercalated in the ensuing multi-molecular structure. The rationale for the design of this novel immunotherapeutic tool and its main features are described in detail in a recent paper [16]. One of the major advances is represented by the use of non-animalderived, ultra-pure, homogeneous low-molecular-weight chondroitin sulfate that represents a different chemical moiety as compared with animal-derived high-molecularweight chondroitin sulfate and shows much-improved efficacy and safety profiles [16]. According to the conclusion of our study "design and development of imuno ${ }^{\circledR}$ represents the culminating point of research in the field of glycosaminoglycans begun in the early eighties as well as the result of years of achievements in the field of immunotherapy with particular reference to GcMAF and Rerum" [16].

Since 2014, the Biomedical Centre for Autism Research and Treatment has accumulated a vast experience with GcMAF [17] and Rerum [15] and, based on the positive outcomes from that experience, we decided to add imuno ${ }^{\circledR}$ to our arsenal of immunotherapeutic approaches. In order to obtain the most objective and complete evaluation of results, we decided to use the Autism Treatment Evaluation Scale also known as Autism Treatment Evaluation Checklist (ATEC), a diagnostic assessment tool that has been demonstrated effective in evaluating interventional effects in autism as well as in following behavioral development over periods of time [18]. This is a major difference compared to the study by Bradstreet et al. on GcMAF [7] and our study on Rerum [15]. In both cases, in-house modified versions of the Clinical Global Impression of Improvement (CGI-I) scale were used. $\mathrm{CGI}-\mathrm{I}$ is a 7 point scale developed to evaluate mental disorders that requires the clinician to assess how much the patient's illness has improved or worsened relative to a baseline state at the beginning of the intervention [19]. The ATEC, however, is a 77-item diagnostic assessment tool where a questionnaire, available in several different languages, is compiled by a parent. At variance with CGI-I, ATEC is specific for autism and evaluates many more areas that are pertinent to the disease such as speech/language and communication (section 1); sociability (section 2); sensory and cognitive awareness (section 3); physical/health behavior (section 4).

In October 2018, the Biomedical Centre for Autism Research and Treatment started using imuno ${ }^{\circledR}$ in autistic subjects. By January 2019, several scores of patients have been treated with this supplement; here, we describe the cases of three autistic patients for whom the treatment was remarkably effective.

\section{Patients and Methods}

At the Biomedical Centre for Autism Research and Treatment, strategies using drugs, when necessary, supplements and nutritional intervention are routinely implemented with the goal of improving symptoms and quality of life of autistic subjects. In addition to pharmacological therapies, nutritional strategies and physical therapy [20,21] tailored to the needs of each individual, a protocol using imuno ${ }^{\circledR}$ has recently been introduced where parents are taught how to administer the supplement to the autistic subjects. imuno ${ }^{\circledR}$ was kindly donated to the Biomedical Centre for Autism Research and Treatment by imuno Corporation, Lini Highway, Port Vila, Vanuatu. The supplement was administered once a week for eight weeks; the dose was calculated taking into account the body weight of the patient. For the cases here described, the dose was $0.05 \mathrm{ml}$ per week. The ATEC questionnaire, in Italian, was compiled by the parents of the patients immediately before starting administration of imuno ${ }^{\circledR}$ and after eight weeks of treatment.

\section{Patients}

Diagnostic criteria for autism adopted at the Biomedical Centre for Autism Research and Treatment are:

1. Marked impairment in the use of multiple nonverbal behaviors such as eye-to-eye gaze, facial expression, body postures, and gestures to regulate social interaction.

2. Failure to develop peer relationships appropriate to developmental level.

3. Lack of spontaneous seeking to share enjoyment, interests or achievements with other people (e.g., by a lack of showing, bringing or pointing out objects of interest).

4. Lack of social or emotional reciprocity.

5. Delay in or total lack of, the development of spoken language.

6. In individuals with adequate speech, marked impairment in the ability to initiate or sustain a conversation with others.

7. Stereotyped and repetitive use of language or idiosyncratic language. 
8. Lack of varied, spontaneous, make-believe play or social imitative play appropriate to developmental level.

9. Encompassing preoccupation with one or more stereotyped and restricted patterns of interest that is abnormal either in intensity or focus.

10. Apparently inflexible adherence to specific nonfunctional routines or rituals.

11. Stereotyped and repetitive motor mannerisms (e.g., hand or finger flapping or twisting, or complex whole-body movements).

\section{Persistent preoccupation with parts of objects.}

The Patients described in this study met six or more criteria from this list and had been diagnosed by either a child neurologist or developmental psychologist, in addition to receiving the evaluation of the clinician at the Biomedical Centre for Autism Research and Treatment.

No significant changes were made to the participants' treatments apart from the introduction of imuno ${ }^{\circledR}$ during the timeframe reported in this study (October 2018-January 2019). The original clinical records, as well as the informed consents given by the patients' parents or legal guardians, are conserved at the Biomedical Centre for Autism Research and Treatment. Since this is a low-number case report (that is, the study describes three or fewer cases) that does not produce generalizable knowledge, nor an investigation of an FDA regulated product, Institutional Review Board (IRB) review is not required for this activity [22].

\section{Results}

\section{Patient 1}

Male, 4 years and 6 months old at the beginning of treatment with imuno ${ }^{\circledR}$. The patient showed the first symptoms of autism around the age of 2 with difficulties in social relationships and stereotypies (spinning of common objects). The patient cries or laughs for no apparent motive and shows difficulties in coordination of movements. According to the parents, he has always suffered from constipation. The patient suffers from absence seizures, confirmed by EEG and treated with valproic acid. After eight weeks of treatment with imuno ${ }^{\circledR}$, the most remarkable improvements were observed for the symptoms listed in Section 4 that is the subscale for physical/health behavior. In particular, the behavioral symptoms described by the queries "bed-wetting", "wets pants/diapers", "soils pants/diapers", "diarrhea", "constipation", "eats too much/too little", "not sensitive to pain" improved from "moderate" to "not a problem", thus indicating complete normalization of these very significant symptoms of autism. The symptom described by the query "sound sensitive" improved from moderate to a minor problem. The symptom described by the query "repetitive movements", another hallmark of autism, improved from serious to minor problem. The symptoms described by the queries "shouts or screams", "demands sameness", "often agitated", all hallmarks of autism, improved from "minor problem" to "not a problem", thus indicating complete normalization of these very significant symptoms. In summary, complete normalization, or a trend toward normalization, of a number of behaviors typical of autism in the area of physical health and behavior were observed.

\section{Patient 2}

Male, 3 years and 9 months old at the beginning of treatment with imuno ${ }^{\circledR}$. The patient showed the first symptoms of autism before the age of 2 with difficulties in deambulation and arrested development. The patient is able to eat only a few types of foods such as yoghurt, milk, homogenized fruit, and small bites of bread. The patient cries or laughs for no apparent motive and, in addition, shows the following symptoms; significant sleep disturbances that are treated with melatonin; chronic constipation; recurrent otitis; hyperactivity/agitation. After eight weeks of treatment with imuno ${ }^{\circledR}$, the most remarkable improvements were observed for the symptoms listed in Section 2 that is the subscale for sociability. In particular, the behavioral symptoms described by the queries "shows no affection", "fails to greet parents", "avoids contact with others", "dislikes being held/cuddled" improved from "very descriptive" to "not descriptive", thus indicating complete normalization of these significant symptoms of autism. The symptoms described by the queries "ignores other people", "pays little attention when addressed", "no eye contact", all hallmarks of autism, improved from "very descriptive" to "somewhat descriptive". In summary, normalization, or a trend toward normalization, of a number of behaviors typical of autism in the area of sociability were observed.

\section{Patient 3}

Male, 3 years and 1 month old at the beginning of treatment with imuno ${ }^{\circledR}$. At one year of age, the patient showed regression of eye contact and, one month later, severe gastroenteritis with high fever, diarrhea and vomiting that led to hospitalization for 10 days. One month after hospitalization, the patient showed loss of strength in his legs. Diarrhea has persisted with up to five episodes per day. The patient laughs or cries with no apparent motive. After eight weeks of treatment with imuno ${ }^{\circledR}$ significant improvements were observed for the symptoms listed in all sections of the ATEC questionnaire. In section 1 that is the subscale for speech/language/communication, improvements were observed for the symptoms described by the queries "responds to no or stops" and "can follow some commands" where the answers showed improvement from "not true" to "somewhat true", and from "somewhat true" to "very true" respectively. In section 2 , that is the subscale for sociability, improvements were observed for the symptoms described by the queries "does not imitate", "disagreeable/not compliant", "in different to being liked" where the answers showed improvement from "somewhat descriptive" to "not descriptive", thus indicating normalization of behaviors. The symptom described by the query "rarely smiles" improved from "very descriptive" to "somewhat descriptive". In section 3 , that is the subscale for sensory/cognitive awareness, improvements were observed for the symptoms described by the queries "looks at people and animals", "plays with toys 
appropriately", "appropriate facial expression", "understand explanations", "initiates activities", "dresses self" where the answers showed improvement from "not descriptive" to "somewhat descriptive", thus indicating a trend toward normalization of behaviors. In section 4, that is the subscale for physical/health behavior, improvements were observed for the symptoms described by the queries "wets pants/ diapers", "shouts or screams", where the answers showed improvement from "minor problem" to "not a problem", thus indicating normalization of these typical autism behaviors. The symptom described by the query "soils pants/diapers" improved from "moderate problem" to "minor problem".

\section{Discussion}

This is the first study where the results of immunotherapy of autism with GcMAF or supplements based on chondroitin sulfate were evaluated using the ATEC, a tool that enables detailed, objective and reliable evaluation of improvements. Our observations indicate that some of the most representative symptoms of autism were completely normalized after eight weeks of treatment and others showed a trend toward normalization. No adverse effects were reported. Since this clinical case report is an open-label, non-controlled, retrospective analysis, caution must be exercised when ascribing cause and effect to any treatment outcome. In addition, the behavioral outcome observation was parentreported as prescribed by the ATEC, and such observation can be biased by parental expectations. It should be noticed, however, that parents of these children had experienced in the past the disappointing outcomes of a number of other treatments and were, therefore, more inclined toward skepticism rather than wishful expectation. Despite these limitations, it appears that the response to imuno ${ }^{\circledR}$ was robust with regard to symptomatic improvements and we attribute these notable results to the effects of the supplement on the immune system and on neuroprotection. It is worth noticing that the cases here described showed different types of responses where the remarkable improvements occurred in different areas of autism. Such a heterogeneity reflects the diversification of responses to treatment, a typical connotation of autism where symptoms and responses to treatment may greatly differ among individuals.

Based on our previous research, we hypothesize that the effects of imuno ${ }^{\circledR}$ on the pathogenetic mechanisms responsible for autism symptoms are related to its effects on the immune system with particular reference to brain lymphatic circulation. In 2015, we published a study supporting the notion that brain lesions and accumulation of extra-axial fluid in the brain of autistic subjects were connected with, and possibly caused by, obstacles in brain lymphatic circulation through the deep cervical nodes [23]. According to our study, infections or inflammatory processes in the mouth, ears, nose, and throat affecting the deep cervical nodes, a quite frequent occurrence, pose an obstacle to drainage of lymph from the brain through the meningeal lymphatic system, also known as glymphatic system. Obstacles in the drainage of lymph from the brain would then lead to the cortical dysplasia and extraaxial fluid accumulation that we had previously documented in the brains of autistic children [24]. Stagnation of lymph in the brain may lead to neuroinflammation that in turn is responsible for autism symptoms [17]. In this context, the observed improvement associated with imuno ${ }^{\circledR}$ treatment may be explained by the effects of the supplement on the flow of lymph at the level of the deep cervical nodes thanks to resolution of inflammation, and by the effects on the endocannabinoid system in analogy with what we had previously demonstrated for GcMAF [17].

From our data, however, imuno ${ }^{\circledR}$ appears to be more potent than GcMAF in improving symptoms of autism. According to Bradstreet et al. [7], improvement of symptoms of autism following GcMAF treatment were observed, on average, after $100 \pm 32$ days with doses ranging from 4 to 100 mg per week, whereas in our observation significant improvements were observed after only eight weeks with a much lower dose. Likewise, the case described by Thyer et al. [8] had been treated with GcMAF for 12 weeks. We attribute this increased efficacy to the presence of low-molecularweight microbial chondroitin sulfate in the composition of imuno ${ }^{\circledR}$. Thus, it has been demonstrated that low-molecularweight microbial chondroitin sulfate is much more potent as far as anti-inflammatory and immune-stimulating properties are concerned [16]. Low-molecular-weight microbial chondroitin sulfate would also be responsible for the effects of imuno ${ }^{\circledR}$ on the endocannabinoid system because of its effects on phospholipase $C$ and arachidonic acid metabolism, an area of research we studied since 1983 [25]. Thus, it was demonstrated that low-molecular-weight species of chondroitin sulfate stimulate phospholipase $C$ [26] with consequent formation of 1,2-diacylglycerol and 1,4,5-inositol trisphosphate. Arachidonic acid-containing 1,2-diacylglycerol is the precursor of 2-arachidonoylglycerol (2-AG) that is the physiological agonist of the two major types of endocannabinoid receptors $C B 1$ and $C B 2$ [27]. Therefore, imuno ${ }^{\circledR}$ by virtue of its effects on $2-A G$, can be considered a valid alternative to plant-derived cannabinoids such as cannabidiol $(C B D)$, in particular when considering that $C B D$ does not act primarily on endocannabinoid receptors but works by enhancing the production of endocannabinoids [28]. Therefore, imuno ${ }^{\circledR}$ by acting directly on physiologic endocannabinoid synthesis would represent a more direct approach to rebalancing the endocannabinoid receptor pathways that are altered in autism $[29,30]$.

In describing these individual cases, we are well aware that these reports, because of their heterogeneity, can be considered anecdotes. However, it is worth noting that a recent study on the evaluation of clinical practice prompts for the re-evaluation of individual cases such as those presented here [31]. Thus, it is well known that some studies present large and impressive statistics obtained from many observations while others report a small number of noteworthy events, as we do in this study. According to the novel, authoritative, epistemological approach, "all of these stories become evidence of what works in medicine" [23] as appears 
to be the case for the reports by Bradstreet et al. and Thyer et al. on the improvement of autism symptoms following GcMAF administration $[7,8]$. Therefore, we suggest that the clinical cases reported here may be considered as evidence for the effectiveness of imuno ${ }^{\circledR}$ in autism and may encourage further studies on this topic.

\section{Acknowledgements}

The Authors wish to thank the parents of the autistic children whose cases are described here for their priceless collaboration. The Authors wish to express their gratitude for the human and scientific legacy of Dr. Bradstreet whose insight inspires their work.

\section{Conflict of Interest}

Nicola Antonucci is the founder of the Biomedical Centre for Autism Research and Treatment, a private clinic. Marco Ruggiero is the inventor of a number of supplements and, together with Stefania Pacini, developed the supplement used in this study. Neither he, nor Dr. Pacini, had any prior knowledge of the therapies being used nor of the details of any patient whose clinical outcomes were being analyzed and described in this study.

\section{Authors' Contribution}

Nicola Antonucci: Performed all the diagnostic and therapeutic procedures described in this study. Marco Ruggiero and Stefania Pacini: Wrote the first draft of this paper, provided critical input and assisted in revising and improving the paper.

\section{Advisory}

No information in this paper is presented by the authors as medical advice. Caregivers, researchers and interested parties should research all information given. Beginning any significant biomedical or other interventions that may impact physiology or making changes to an established regimen should be discussed with the patient's physician in advance. Standard of care for each pathology must be followed as well as rules and regulations established by Health Authorities of each Country.

\section{References}

1. Ruggiero M, Pacini S. From neurology to oncology: what have in common autism and cancer? the role of oncogenes, immune system and microbiota. J Neurol Stroke. 2018; 8(3): 166-172. doi: 10.15406/ jnsk.2018.08.00303

2. Inui T, Kuchiike D, Kubo K, et al. Clinical experience of integrative cancer immunotherapy with GcMAF. Anticancer Res. 2013; 33(7): 2917-2919.

3. Thyer L, Ward E, Smith R, et al. GC protein-derived macrophage-activating factor decreases $\alpha-N$-acetylgalactosaminidase levels in advanced cancer patients. Oncoimmunology. 2013; 2(8): e25769. doi: 10.4161/onci.25769

4. Ruggiero $M$, Ward $E$, Smith $R$, et al. Oleic Acid, deglycosylated vitamin $D$-binding protein, nitric oxide: a molecular triad made lethal to cancer. Anticancer Res. 2014; 34(7): 3569-3578.
5. BrancaJJ, Pacini S, Ruggiero M. Effects of Pre-surgical Vitamin D Supplementation and Ketogenic Diet in a Patient with Recurrent Breast Cancer. Anticancer Res. 2015; 35(10): 5525-32.

6. Inui T, Amitani H, Kubo K, et al. Case Report: A Non-small Cell Lung Cancer Patient Treated with GcMAF, Sonodynamic Therapy and Tumor Treating Fields. Anticancer Res. 2016; 36(7): 3767-3770.

7. Bradstreet JJ, Vogelaar E, Thyer L. Initial Observations of Elevated Alpha$\mathrm{N}$-Acetylgalactosaminidase Activity Associated with Autism and Observed Reductions from GC Protein-Macrophage Activating Factor Injections. Autism Insights. 2012; 4: 31-38. doi: 10.4137/AUI.S10485

8. Thyer L, Ward E, Smith $R$, et al. Therapeutic effects of highly purified deglycosylated GCMAF in the immunotherapy of patients with chronic diseases. Am J Immunol. 2013; 9(3): 78-84. doi: 10.3844/ajisp.2013.78.84

9. Morucci G, Branca JJV, Gulisano M, et al. Gc-protein-derived macrophage activating factor counteracts the neuronal damage induced by oxaliplatin. Anticancer Drugs. 2015; 26(2): 197-209. doi: 10.1097/CAD.0000000000000177

10. Branca JJV, Morucci G, Malentacchi F, Gelmini S, Ruggiero M, Pacini S. Effects of oxaliplatin and oleic acid Gc-protein-derived macrophageactivating factor on murine and human microglia. J Neurosci Res. 2015; 93(9): 1364-1377. doi: 10.1002/jnr.23588

11. Salter MW, Stevens B. Microglia emerge as central players in brain disease. Nat Med. 2017; 23(9): 1018-1027. doi: 10.1038/nm.4397

12. Pacini S, Morucci G, Punzi T, Gulisano M, Ruggiero M. Gc protein-derived macrophage-activating factor (GCMAF) stimulates cAMP formation in human mononuclear cells and inhibits angiogenesis in chick embryo chorionallantoic membrane assay. Cancer Immunol Immunother. 2011; 60(4): 479-485. doi: 10.1007/s00262-010-0953-7

13. Borges CR, Rehder DS. Glycan structure of Gc Protein-derived Macrophage Activating Factor as revealed by mass spectrometry. Arch Biochem Biophys. 2016; 606: 167-79. doi: 10.1016/j.abb.2016.08.006

14. Ruggiero $M$, Reinwald $H$, Pacini $S$. Is chondroitin sulfate responsible for the biological effects attributed to the GC protein-derived Macrophage Activating Factor (GcMAF)? Med Hypotheses. 2016; 94: 126-131. doi: 10.1016/j.mehy.2016.07.012

15. Antonucci N, Pacini S, Ruggiero M. Clinical Case Report of a Novel Immunotherapeutic Approach to Autism Spectrum Disorders based on an emulsion of Chondroitin Sulfate, Vitamin D3 and Oleic Acid. Am J Immunol. 2017; 13(3): 180-185. doi: 10.3844/ajisp.2017.180.185

16. Ruggiero $M$, Pacini $S$. Rationale for the design of a novel tool for immunotherapy based on an emulsion of glycosaminoglycan. Integr Cancer Sci Therap. 2018; 5: 1-5. doi: 10.15761/ICST.1000285

17. Siniscalco $D$, Bradstreet JJ, Cirillo $A$, Antonucci N. The in vitro GcMAF effects on endocannabinoid system transcriptionomics, receptor formation, and cell activity of autism-derived macrophages. J Neuroinflammation. 2014; 11: 78. doi: 10.1186/1742-2094-11-78

18. Geier DA, Kern JK, Geier MR. A Comparison of the Autism Treatment Evaluation Checklist (ATEC) and the Childhood Autism Rating Scale (CARS) for the Quantitative Evaluation of Autism. J Ment Health Res Intellect Disabil. 2013; 6(4): 255-267. doi: 10.1080/19315864.2012.681340

19. Dieltjens M, Verbraecken JA, Hedner J, et al. Use of the Clinical Global Impression scale in sleep apnea patients - Results from the ESADA database. Sleep Med. 2018; 9457(18): 30370-30380. doi: 10.1016/.j.sleep.2018.10.028

20. Antonucci N, Cirillo A, Siniscalco D. Beneficial effects of palmitoylethanolamide on expressive language, cognition, and behaviors in autism: A report of two cases. Case Rep Psychiatry. 2015; 2015: 325061. doi: 10.1155/2015/325061

21. Antonucci N, Pacini S, Ruggiero M. Clinical Experience of Integrative Autism Treatment with Manual Lymphatic Drainage. EC Neurol. 2019; 11 21-28.

22. John Hopkins Medicine. 102.3 Organization Policy on Single Case Reports and Case Series. 2019.

23. Bradstreet JJ, Ruggiero M, Pacini S. Commentary: Structural and functional features of central nervous system lymphatic vessels. Front Neurosci. 2015; 9: 485. doi: 10.3389/fnins.2015.00485 
24. Bradstreet JJ, Pacini S, Ruggiero M. A New Methodology of Viewing ExtraAxial Fluid and Cortical Abnormalities in Children with Autism via Transcranial Ultrasonography. Front Hum Neurosci. 2014; 7: 934. doi: 10.3389/fnhum.2013.00934

25. Chiarugi V, Ruggiero M, Ricoveri W. Rapid and simple method for the detection of nanogram amounts of arachidonate metabolites resolved by thin-layer chromatography. J Chromatogr. 1983; 280: 400-403. doi: 10.1016/S0021-9673(00)91585-1

26. Sotogaku N, Tully $\mathrm{SE}$, Gama $\mathrm{Cl}$, et al. Activation of phospholipase $\mathrm{C}$ pathways by a synthetic chondroitin sulfate- $E$ tetrasaccharide promotes neurite outgrowth of dopaminergic neurons. J Neurochem. 2007; 103(2): 749-760. doi: 10.1111/j.1471-4159.2007.04849.x

27. Sugiura $T$, Kodaka $T$, Nakane $S$, et al. Evidence that the cannabinoid CB1 receptor is a 2-arachidonoylglycerol receptor. Structure-activity relationship of 2-arachidonoylglycerol, ether-linked analogues, and related compounds. J Biol Chem. 1999; 274(5): 2794-2801.
28. Campos AC, Moreira FA, Gomes FV, Del Bel EA, Guimarães FS. Multiple mechanisms involved in the large-spectrum therapeutic potential of cannabidiol in psychiatric disorders. Philos Trans R Soc Lond B Biol Sci. 2012; 367(1607): 3364-3378. doi: 10.1098/rstb.2011.0389

29. Chakrabarti B, Persico A, Battista N, Maccarrone M. Endocannabinoid Signaling in Autism. J Neurotherapeutics. 2015; 12: 837-847. doi: 10.1007/ s13311-015-0371-9

30. Zamberletti E, Gabaglio M, Parolaro D. The Endocannabinoid System and Autism Spectrum Disorders: Insights from Animal Models. Int J Mol Sci. 2017; 18(9): 1916. doi: 10.3390/ijms18091916

31. Nunn R. Mere anecdote: evidence and stories in medicine. J Eval Clin Pract. 2011; 17: 920-926. doi: 10.1111/j.1365-2753.2011.01727.x 